\title{
Geometric Entanglement of Symmetric States and the Majorana Representation
}

\author{
Martin Aulbach, 1,2,* Damian Markham, 3, 丹 and Mio Murao ${ }^{4,5}$, 田 \\ ${ }^{1}$ The School of Physics and Astronomy, University of Leeds, Leeds LS2 9JT, United Kingdom \\ ${ }^{2}$ Department of Physics, University of Oxford, Clarendon Laboratory, Oxford OX1 3PU, United Kingdom \\ ${ }^{3}$ CNRS, LTCI, Telecom ParisTech, 23 Avenue d'Italie, 75013 Paris, France \\ ${ }^{4}$ Department of Physics, Graduate School of Science, \\ The University of Tokyo, Tokyo 113-0033, Japan \\ ${ }^{5}$ Institute for Nano Quantum Information Electronics, \\ The University of Tokyo, Tokyo 113-0033, Japan
}

\begin{abstract}
Permutation-symmetric quantum states appear in a variety of physical situations, and they have been proposed for quantum information tasks. This article builds upon the results of [New J. Phys. 12, 073025 (2010)], where the maximally entangled symmetric states of up to twelve qubits were explored, and their amount of geometric entanglement determined by numeric and analytic means. For this the Majorana representation, a generalization of the Bloch sphere representation, can be employed to represent symmetric $n$ qubit states by $n$ points on the surface of a unit sphere. Symmetries of this point distribution simplify the determination of the entanglement, and enable the study of quantum states in novel ways. Here it is shown that the duality relationship of Platonic solids has a counterpart in the Majorana representation, and that in general maximally entangled symmetric states neither correspond to anticoherent spin states nor to spherical designs. The usability of symmetric states as resources for measurement-based quantum computing is also discussed.
\end{abstract}

Keywords: Majorana representation, geometric measure, symmetric, entanglement, anticoherent, spherical design

\section{INTRODUCTION}

Multipartite entanglement is a crucial resource for many tasks in quantum information science, but its quantification is difficult due to the existence of different types of entanglement [1]. It is therefore unsurprising that many different entanglement measures have been proposed in order to quantify the amount of entanglement of multipartite quantum states [2]. Here we build upon our results about highly and maximally entangled permutation-symmetric quantum states in terms of the geometric measure of entanglement [3]. This restriction to a subset of quantum states - studied under a particular entanglement measure - makes it possible to gain strong results [3, 4], and to find a rare visual representation of multipartite entanglement.

Permutation-symmetric quantum states are invariant under any permutation of their subsystems. Such states appear in many-body physics, and they have found use in leader election [5]. Furthermore, they have been actively implemented experimentally [6, 7], and their symmetric properties facilitate the analysis of entanglement [8-13]. In order to analyze the usefulness of symmetric states for measurement-based quantum computation (MBQC) [14], the geometric measure of entanglement is particularly suited, because the classification of states as MBQC-resources has been performed in terms of this measure [15 17$]$.

\footnotetext{
* m.aulbach1@physics.ox.ac.uk

$\dagger$ markham@telecom-paristech.fr

$\ddagger$ murao@phys.s.u-tokyo.ac.jp
}

The central tool for our analysis of symmetric entanglement is the Majorana representation [18], a generalization of the Bloch sphere representation of single qubits. By means of this representation any $n$ qubit symmetric state can be unambiguously mapped to $n$ points on the surface of the unit sphere. Recently the Majorana representation has been used to search for and characterize different classes of SLOCC entanglement [8, 11, 12, 19], which is related to the classification of phases in spinor condensates [11, 20]. It has also been employed to search for the "least classical" state of a spin- $j$ system [21], and the solutions of this problem are intimately related to the maximally entangled symmetric states. Furthermore, the Majorana representation has been used for the study of spherical designs [22], Berry phases in high spin systems [23], quantum chaos [24, 25], optimal resources for reference frame alignment 26], phase estimation [27], phases in spinor BEC 20, 28], classicality in terms of the discriminability of states [29], for finding solutions to the Lipkin-Meshkov-Glick model [30] and for finding efficient proofs of the Kochen-Specker theorem [31].

The article is organized as follows: In Section II we briefly recapitulate the geometric measure of entanglement. This is followed by Section III where the geometric entanglement of permutation-symmetric states and its implications for MBQC is discussed. In Section IV the Majorana representation is introduced for symmetric states of $n$ qubits, which is followed by Section $\nabla$ which reviews our analytical and numerical findings that we recently published in [3]. In Section VI the usefulness of the Majorana representation is demonstrated for highly entangled symmetric states whose point distributions are described by Platonic solids. The entanglement of such states is particularly easy to determine with the known 
theoretical results, and it is found that there exists an intriguing analogy with the dual polyhedra of the Platonic solids 32]. Anticoherent spin states [33] and the mathematical concept of spherical designs [22] are briefly mentioned, and it is shown that in general the maximally entangled symmetric states do not represent anticoherent states or spherical designs. Finally, SectionVII concludes this article with a summary of our results.

\section{GEOMETRIC MEASURE OF ENTANGLEMENT}

The geometric measure of entanglement is a distancelike entanglement measure in the sense that it assesses the entanglement in terms of the remoteness from the set of separable states [34]. It is defined as the maximal overlap of a normalized pure state with all normalized pure product states $35-37$.

$$
E_{\mathrm{g}}(|\psi\rangle)=\min _{|\lambda\rangle \in \mathcal{H}_{\mathrm{SEP}}}-\log _{2}|\langle\lambda \mid \psi\rangle|^{2}
$$

A product state closest to $|\psi\rangle$ is denoted by $\left|\Lambda_{\psi}\right\rangle$, and it should be kept in mind that a given $|\psi\rangle$ can have more than one closest product state. The problem of maximizing the entanglement can be written as a max-minproblem:

$$
\begin{aligned}
E_{\mathrm{g}}^{\max } & =\max _{|\psi\rangle \in \mathcal{H}} \min _{|\lambda\rangle \in \mathcal{H}_{\mathrm{SEP}}}-\log _{2}|\langle\lambda \mid \psi\rangle|^{2} \\
& =\max _{|\psi\rangle \in \mathcal{H}}-\log _{2}\left|\left\langle\Lambda_{\psi} \mid \psi\right\rangle\right|^{2}=-\log _{2}\left|\left\langle\Lambda_{\Psi} \mid \Psi\right\rangle\right|^{2} .
\end{aligned}
$$

The geometric measure is closely related to the robustness of entanglement $R$ [38] and the relative entropy of entanglement $E_{\mathrm{R}}$ [34], two other distance-like entanglement measures. The inequalities $E_{\mathrm{g}} \leq E_{\mathrm{R}} \leq \log _{2}(1+R)$ hold for all states [9, 39, 40], and they become equalities for stabilizer states, Dicke states and permutationantisymmetric basis states [9, 41, 42]. Some advantages of the geometric measure are its comparatively easy calculation, its applications in related fields of physics $43-$ 45], and its operational interpretations, e.g. in local state discrimination [41], additivity of channel capacities [46] and for the classification of states as resources for measurement-based quantum computation (MBQC) 15 17].

A general quantum state of a finite-dimensional system can be cast as $|\psi\rangle=\sum_{i} a_{i}|i\rangle$ with complex coefficients $a_{i}$ and an orthonormal basis $\{|i\rangle\}$. The state $|\psi\rangle$ is called real if (for a given basis) the $a_{i}$ are all real, and positive if the $a_{i}$ are all positive. Every positive state $|\psi\rangle$ has at least one positive closest product state $\left|\Lambda_{\psi}\right\rangle$ [3, 47], a result which simplifies the determination of their entanglement.

\section{PERMUTATION-SYMMETRIC STATES}

Permutation-symmetric quantum states are states that are invariant under any permutation of their subsystems, i.e. $P|\psi\rangle=|\psi\rangle$ for all $P \in S_{N}$. For $n$ qubits the Hilbert space of symmetric states is spanned by the Dicke states, the equally weighted sums of all permutations of computational basis states with $n-k$ qubits being $|0\rangle$ and $k$ being $|1\rangle$ [48, 49].

$$
\left|S_{n, k}\right\rangle=\left(\begin{array}{l}
n \\
k
\end{array}\right)^{-1 / 2} \sum_{\text {perm }} \underbrace{|0\rangle|0\rangle \cdots|0\rangle}_{n-k} \underbrace{|1\rangle|1\rangle \cdots|1\rangle}_{k},
$$

with $0 \leq k \leq n$. A general pure symmetric state of $n$ qubits is a linear combination of the $n+1$ symmetric basis states $\left|S_{n, k}\right\rangle$. We will abbreviate this notation to $\left|S_{k}\right\rangle$ whenever the number of qubits is clear.

It was recently found that all closest product states of multipartite ( $\geq 3$ parts) symmetric states are symmetric themselves, and that bipartite symmetric states have at least one symmetric closest product state [10]. Furthermore, it can be shown that positive symmetric states have at least one positive symmetric closest product state [9]. These results considerably reduce the complexity of finding the closest product state and thus the entanglement of a symmetric state.

The theoretical and experimental analysis of symmetric state entanglement, e.g. as entanglement witnesses or in experimental setups [6, 77, 50, 51], is valuable, because symmetric states appear in many-body physics. For example, the ground state of the Lipkin-MeshkovGlick model is permutation-invariant, and its entanglement has been quantified in term of the geometric measure [52].

\section{A. Bounds on Maximal Entanglement}

In this subsection we will briefly discuss the known upper and lower bounds on the maximal possible amount of geometric entanglement. It should however be kept in mind that the maximally entangled state and its amount of entanglement depends on the chosen entanglement measure [53].

The maximal possible entanglement of general $n$ qubit states scales linearly with the number of qubits, namely

$$
\frac{n}{2} \leq E_{\mathrm{g}}^{\max } \leq n-1 .
$$

The left-hand side of the inequality is clear from the trivial example of an $n$ qubit state ( $n$ even) composed of $\frac{n}{2}$ bipartite Bell states, or from 2D cluster states [42]. The upper bound was derived in 54]. It is also known that most $n$ qubit states are much closer to the upper bound than to the lower bound. More precisely, for $n>10$ qubits the overwhelming majority of states have entanglement $E_{\mathrm{g}}>n-2 \log _{2}(n)-3[15]$. 
For symmetric states a trivial lower bound can be derived from the Dicke states. A closest product state of $\left|S_{n, k}\right\rangle$ is known [9] to be

$$
|\Lambda\rangle=\left(\sqrt{\frac{n-k}{n}}|0\rangle+\sqrt{\frac{k}{n}}|1\rangle\right)^{\otimes n} .
$$

From this the entanglement follows as

$$
E_{\mathrm{g}}\left(\left|S_{n, k}\right\rangle\right)=\log _{2}\left(\frac{\left(\frac{n}{k}\right)^{k}\left(\frac{n}{n-k}\right)^{n-k}}{\left(\begin{array}{l}
n \\
k
\end{array}\right)}\right) .
$$

The maximally entangled Dicke state is $\left|S_{n, n / 2}\right\rangle$ for even $n$ and the two equivalent states $\left|S_{n,\lfloor n / 2\rfloor}\right\rangle$ and $\left|S_{n,\lceil n / 2\rceil}\right\rangle$ for odd $n$. Their Stirling approximation for large $n$ yields $E_{\mathrm{g}}^{\max } \geq \log _{2} \sqrt{n \pi / 2}$. An upper bound to the geometric measure of symmetric $n$ qubit states has been derived from the decomposition of the identity on symmetric subspace, yielding $E_{\mathrm{g}}^{\max } \leq \log _{2}(n+1)$, see e.g. [55]. An alternative proof with the benefit of being visually accessible by means of the Majorana representation will be given in Theorem 2 .

Combining these bounds, it is seen that the maximal symmetric entanglement of $n$ qubits scales as

$$
\log _{2} \sqrt{\frac{n \pi}{2}} \leq E_{\mathrm{g}}^{\max } \leq \log _{2}(n+1),
$$

i.e. polylogarithmically between $\mathcal{O}(\log \sqrt{n})$ and $\mathcal{O}(\log n)$. Numerical evidence suggests that the actual values are much closer to the upper bound than to the lower bound, and $E_{\mathrm{g}}^{\max } \gtrsim \log _{2}(n+1)-0.775$ can be considered a reliable lower bound [4].

\section{B. Resources for MBQC}

We have seen that the maximal entanglement of symmetric states scales much slower than that of general states, namely logarithmically rather than linearly. This need not be a disadvantage for symmetric states, though, and in fact could render them useful for MBQC [14], because it was shown that if the entanglement of a state is too large, then it cannot be a good resource for MBQC. More specifically, if the $n$ qubit entanglement scales larger than $n-\delta$ for some constant $\delta$, then such a computation can be simulated efficiently classically [15]. This rules out many general quantum states as MBQC resources, but not symmetric ones.

On the other hand, universal resources for MBQC must be maximally entangled in a certain sense [16, 17]. Considering the qualitative departure of the scaling relation (17) from (4), it is questionable whether symmetric states are sufficiently entangled to be MBQC resources. Indeed, permutation-symmetric states can be ruled out as exact, deterministic MBQC resources, because their entanglement does not scale faster-than-logarithmically [3, 17]. Somewhat weaker requirements are imposed upon approximate, stochastic MBQC resources [16], although this generally leads only to a small extension of the class of suitable resources in the vicinity of exact, deterministic resources (e.g. 2D cluster states with holes). It is therefore believed that symmetric states cannot be used even for approximate, stochastic MBQC.

As an example, we will show that Dicke states with a fixed number of excitations cannot be useful for $\epsilon$ approximate, deterministic MBQC [16]. Roughly speaking, $\epsilon$-approximate universal resource states can be converted into any other state by LOCC with an inaccuracy of at most $\epsilon$. The $\epsilon$-version of the geometric measure [56] is defined as 16 .

$$
E_{\mathrm{G}}^{\epsilon}(\rho)=\min \left\{E_{\mathrm{G}}(\sigma) \mid D(\rho, \sigma) \leq \epsilon\right\},
$$

where $D$ is a distance that is "strictly related to the fidelity", meaning that for any two states $\rho$ and $\sigma$, $D(\rho, \sigma) \leq \epsilon \Rightarrow F(\rho, \sigma) \geq 1-\eta(\epsilon)$, where $0 \leq \eta(\epsilon) \leq 1$ is a strictly monotonically increasing function with $\eta(0)=0$. $E_{\mathrm{G}}^{\epsilon}(\rho)$ can be understood as the guaranteed entanglement obtained from a preparation of $\rho$ with inaccuracy $\epsilon$. One possible choice of $D$ is the trace distance, which for pure states reads $D_{\mathrm{t}}(|\psi\rangle,|\phi\rangle)=\sqrt{1-|\langle\psi \mid \phi\rangle|^{2}}=$ $\sqrt{1-F}$, where $F$ is the fidelity. In this case one can choose $\eta(\epsilon)=\epsilon^{2}$.

As shown in Example 1 of [16], the family of W states $\Psi_{\mathrm{W}}=\left\{\left|\mathrm{W}_{n}\right\rangle\right\}_{n}$, with $\left|\mathrm{W}_{n}\right\rangle \equiv\left|S_{n, 1}\right\rangle$, is not an $\epsilon$ approximate universal resource for $\eta(\epsilon) \lesssim 0.001$. This result can be generalized to all families of Dicke states $\Psi_{S_{k}}=\left\{\left|S_{n, k}\right\rangle\right\}_{n}$ with a fixed number of excitations $k$.

Example 1. For any fixed $k \in \mathbb{N}$ the family of Dicke states $\Psi_{S_{k}}=\left\{\left|S_{n, k}\right\rangle\right\}_{n}$ cannot be an $\epsilon$-approximate universal $M B Q C$ resource for $\eta(\epsilon) \lesssim 0.001 k^{-3 / 2}$.

Proof. Using Equation (6) and the Stirling approximation for high $n$, the asymptotic geometric entanglement of the family $\Psi_{S_{k}}$ is found to be

$$
E_{\mathrm{G}}\left(\Psi_{S_{k}}\right)=1-\frac{k^{k}}{\mathrm{e}^{k} k !}
$$

Specifically, the amount of geometric entanglement remains finite for arbitrary values of $n$, allowing us to apply Proposition 3 and Theorem 1 of [16] to show that the necessary condition for $\epsilon$-approximate deterministic universality,

$$
E_{\mathrm{G}}\left(\Psi_{S_{k}}\right)>1-4 \eta^{1 / 3}+3.4 \eta^{2 / 3}
$$

is violated for $\eta(\epsilon) \lesssim 0.001 k^{-3 / 2}$.

Of course, it should be noted that many other quantum information tasks are not restricted by the requirements of MBQC-universality, and that highly entangled symmetric states can therefore be valuable resources for such tasks. 


\section{MAJORANA REPRESENTATION OF SYMMETRIC STATES}

The classical angular momentum $\mathbf{J}$ of a physical system can be represented by a single point on the surface of the unit sphere in $\mathbb{R}^{3}$, corresponding to the direction of J. Quantum mechanics does not allow for such a simple representation, but it is possible to uniquely represent a pure state of spin- $j$ by $2 j$ undistinguishable points on the sphere [18]. This is a generalization of the Bloch sphere representation of a qubit. An equivalent representation can be shown to exist for symmetric states of $n$ spin$(1 / 2)$ particles [18, 57], with an isomorphism mediating between all states of a spin- $j$ particle and the symmetric states of $2 j$ qubits.

Hence, this "Majorana representation" allows us to uniquely compose any symmetric state of $n$ qubits $|\psi\rangle_{\mathrm{s}}$ from a sum over all permutations $P: S_{N} \rightarrow S_{N}$ of $n$ undistinguishable qubits $\left\{\left|\phi_{1}\right\rangle, \ldots,\left|\phi_{n}\right\rangle\right\}$ :

$$
\begin{gathered}
|\psi\rangle_{\mathrm{s}}=K^{-1 / 2} \sum_{\text {perm }}\left|\phi_{P(1)}\right\rangle\left|\phi_{P(2)}\right\rangle \cdots\left|\phi_{P(n)}\right\rangle, \text { with } \\
\left|\phi_{i}\right\rangle=\cos \frac{\theta_{i}}{2}|0\rangle+\mathrm{e}^{\mathrm{i} \varphi_{i}} \sin \frac{\theta_{i}}{2}|1\rangle
\end{gathered}
$$

and where the normalization factor $K$ depends on the given state. The identity (11) allows the visualization of the multi-qubit state $|\psi\rangle_{\mathrm{s}}$ by $n$ points on a sphere. In the following these points will be called the Majorana points (MP), and the sphere on which they lie the Majorana sphere.

As outlined in the previous section, for $n \geq 3$ qubits every closest product state $|\Lambda\rangle$ of a symmetric state $|\psi\rangle_{\mathrm{s}}$ is symmetric itself [10], and can therefore be written as $|\Lambda\rangle=|\sigma\rangle^{\otimes n}$, with a single qubit state $|\sigma\rangle$. The closest product states of a given symmetric state can therefore be visualized by Bloch vectors too, and in analogy to the Majorana points, we call $|\sigma\rangle$ a closest product point (CPP).

For symmetric states the scalar product from the definition of the geometric measure can be concisely expressed in terms of the MPs and a CPP:

$$
\left|\langle\Lambda \mid \psi\rangle_{\mathrm{s}}\right|=n ! K^{-1 / 2} \prod_{i=1}^{n}\left|\left\langle\sigma \mid \phi_{i}\right\rangle\right| .
$$

To determine the CPP of a given symmetric state, one therefore has to maximize the absolute value of a product of scalar products. The factors $\left\langle\sigma \mid \phi_{i}\right\rangle$ are the angles between the corresponding Bloch vectors on the Majorana sphere, thus turning the determination of the CPP into a geometrical optimization problem.

From Equation (11) it follows that the application on an arbitrary single-qubit unitary operation $U$ to each of the $n$ subsystems of a symmetric state $|\psi\rangle_{\mathrm{s}}$ yields

$$
\begin{aligned}
|\varphi\rangle_{\mathrm{s}} & =U^{\otimes n}|\psi\rangle_{\mathrm{s}} \\
& =K^{-1 / 2} \sum_{\text {perm }}\left(U\left|\phi_{P(1)}\right\rangle\right) \otimes \cdots \otimes\left(U\left|\phi_{P(n)}\right\rangle\right) .
\end{aligned}
$$

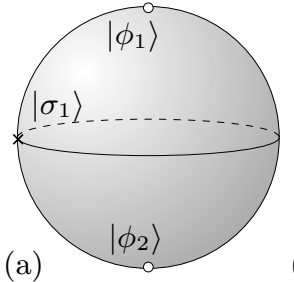

(a)

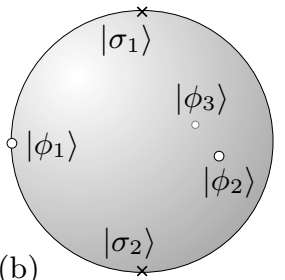

(b)

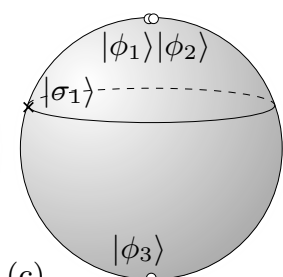

(c)

FIG. 1. Majorana representations of symmetric states of two and three qubits. MPs are depicted as white dots and CPPs as crosses or dashed lines. The pictures show (a) the two qubit Bell state $\left|\psi^{+}\right\rangle$, (b) three qubit GHZ state and (c) three qubit W state.

Thus the symmetric state $|\psi\rangle_{\mathrm{S}}$ is mapped to a symmetric state $|\varphi\rangle_{\mathrm{s}}$ whose MP distribution can be obtained from a joint rotation of the MPs of $|\psi\rangle_{\mathrm{s}}$ along a common axis on the Majorana sphere. The two LOCC-equivalent states $|\psi\rangle_{\mathrm{S}}$ and $|\varphi\rangle_{\mathrm{S}}$ have the same relative MP distribution, and therefore the same number and relative distribution of CPPs, as well as the same amount of entanglement.

\section{A. Examples}

For pure symmetric states of two qubits the only absolute degree of freedom in the Majorana representation (and hence entanglement) is the distance between the two MPs. It is easy to determine that the CPP lies halfway between the two MPs, and that the entanglement is maximized when the MPs lie antipodal to each other. Figure 1 (a) shows the Bell state $\left|\psi^{+}\right\rangle=1 / \sqrt{2}(|01\rangle+|10\rangle)$ with its two MPs $\left|\phi_{1}\right\rangle=|0\rangle$ and $\left|\phi_{2}\right\rangle=|1\rangle$. Due to this azimuthal symmetry the CPPs form a continuous ring $|\sigma\rangle=1 / \sqrt{2}\left(|0\rangle+\mathrm{e}^{\mathrm{i} \varphi}|1\rangle\right)$, with $\varphi \in[0,2 \pi)$ around the equator. The amount of entanglement is $E_{\mathrm{g}}\left(\left|\psi^{+}\right\rangle\right)=1$.

For three qubits the GHZ state and W state, two positive symmetric states, are considered to be extremal [58], with the $\mathrm{W}$ state proven to be the maximally entangled state in terms of the geometric measure [59].

The MPs of the tripartite GHZ state $|\mathrm{GHZ}\rangle=$ $1 / \sqrt{2}(|000\rangle+|111\rangle)$ are, up to normalization,

$$
\begin{aligned}
& \left|\phi_{1}\right\rangle=|0\rangle+|1\rangle, \\
& \left|\phi_{2}\right\rangle=|0\rangle+\mathrm{e}^{\mathrm{i} 2 \pi / 3}|1\rangle, \\
& \left|\phi_{3}\right\rangle=|0\rangle+\mathrm{e}^{\mathrm{i} 4 \pi / 3}|1\rangle .
\end{aligned}
$$

Its two CPPs are $\left|\sigma_{1}\right\rangle=|0\rangle$ and $\left|\sigma_{2}\right\rangle=|1\rangle$, and the amount of entanglement is $E_{\mathrm{g}}(|\mathrm{GHZ}\rangle)=1$. Figure 1(b) shows the Majorana representation of the GHZ state. The three MPs form an equilateral triangle on the equator, and the two CPPs are the north pole and south pole.

In the case of the $\mathrm{W}$ state $|\mathrm{W}\rangle=\left|S_{3,1}\right\rangle=$ $1 / \sqrt{3}(|001\rangle+|010\rangle+|100\rangle)$, a Dicke state, the MPs can be directly accessed from its definition as $\left|\phi_{1}\right\rangle=\left|\phi_{2}\right\rangle=$ $|0\rangle$ and $\left|\phi_{3}\right\rangle=|1\rangle$. The positive CPP follows from Equation (5) as $\left|\sigma_{1}\right\rangle=\sqrt{2 / 3}|0\rangle+\sqrt{1 / 3}|1\rangle$, and the azimuthal 
symmetry implies that the set of all CPPs is formed by $|\sigma\rangle=\sqrt{2 / 3}|0\rangle+\mathrm{e}^{\mathrm{i} \varphi} \sqrt{1 / 3}|1\rangle$, with $\varphi \in[0,2 \pi)$. The Majorana representation is shown in Figure 1(c), and the entanglement is $E_{\mathrm{g}}(|\mathrm{W}\rangle)=\log _{2}(9 / 4) \approx 1.17$.

\section{B. Extremal Point Distributions}

With Equation (12) the min-max-problem (2) of finding the maximally entangled symmetric state can be recast as

$$
\min _{\left\{\left|\phi_{i}\right\rangle\right\}} K^{-1 / 2}\left(\max _{|\sigma\rangle} \prod_{i=1}^{n}\left|\left\langle\sigma \mid \phi_{i}\right\rangle\right|\right) .
$$

Solving this "Majorana problem" is far from trivial, particularly with the normalization factor $K$ depending on the MPs. The problem can be understood as an optimization problem on the sphere, prompting the question whether the known solutions of classical point distribution problems on the sphere [60] can help in finding the solutions of the Majorana problem. Two problems that have been extensively studied in the past are Tóth's problem and Thomson's problem.

Tóth's problem states that $n$ points have to be distributed over the sphere so that the minimum pairwise distance becomes maximal [60]. Point configurations that solve this problem are known as spherical codes or sphere packings.

Thomson's problem is considering $n$ point charges which are confined to the surface of a sphere and interacting with each other through Coulomb's inverse square law. The desired distribution is the one which minimizes the potential energy [61]. This problem has a variety of applications, e.g. for multi-electron bubbles in liquid Helium 62], liquid metal drops confined in Paul traps 63], shell structure of viruses 64], colloidosomes 65], fullerene patterns [66] and Abrikosov lattice of vortices in superconducting metal shells [67].

Exact solutions to Tóth's and Thomson's problem of $n$ points are known only for very few and low $n$ 60, 68], but numerical solutions are known for a much wider range of $n$ in both problems [69, 70]. An illustrating example are the five Platonic solids - the regular convex polyhedra whose edges, vertices and angles are all congruent, see Figure 2. Because of their high symmetry one would expect that their vertices solve Tóth's and Thomson's problem for the corresponding $n$. This is however true only for $n=4,6,12$, but not for $n=8,20$.
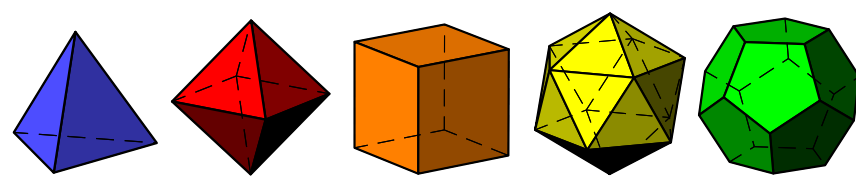

FIG. 2. (color online) The five Platonic solids from left to right: tetrahedron $(n=4)$, octahedron $(n=6)$, cube $(n=8)$, icosahedron $(n=12)$, and dodecahedron $(n=20)$.
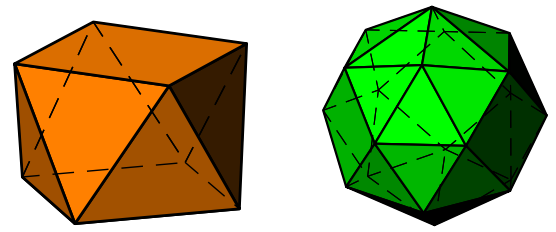

FIG. 3. (color online) For $n=8$ the solution of Tóth's problem is given by a cubic antiprism, and for $n=20$ by a polyhedron consisting of 30 triangles and 3 rhombuses.

Figure 3 depicts the polyhedra that solve Tóth's problem for $n=8,20$. For $n=8$ the solution is the cubic antiprism, which can be obtained from the cube by rotating one face by 45 degrees, followed by a slight compression along the direction perpendicular to the rotated face. In this way, the nearest neighbor distances between the vertices can be equally reduced, at the expense of breaking the high Platonic symmetry. This simple example shows that it is in general not easy to find the optimal spherical distribution for a set of points, and this is also true for the Majorana problem.

\section{ANALYTIC RESULTS ABOUT MPS AND CPPS}

This section summarizes the analytic results about the Majorana representation that we have presented in [3]. In particular, the relationship between the coefficients of a symmetric state $|\psi\rangle_{\mathrm{s}}=\sum_{k=0}^{n} a_{k}\left|S_{k}\right\rangle$ and the corresponding distribution of MPs and CPPs on the Majorana sphere will be illuminated.

Theorem 2. For every symmetric $n$ qubit state $|\psi\rangle_{s}$ the following holds:

$$
\int_{0}^{2 \pi} \int_{0}^{\pi}\left|\langle\lambda(\theta, \varphi) \mid \psi\rangle_{s}\right|^{2} \sin \theta \mathrm{d} \theta \mathrm{d} \varphi=\frac{4 \pi}{n+1},
$$

where $|\lambda(\theta, \varphi)\rangle=\left(\cos \frac{\theta}{2}|0\rangle+\mathrm{e}^{\mathrm{i} \varphi} \sin \frac{\theta}{2}|1\rangle\right)^{\otimes n}$.

For the proof of this theorem we refer to [3]. The remarkable property of Equation (16) is that the integral is the same for all symmetric $n$ qubit states, thus straightforwardly yielding the upper bound $E_{\mathrm{g}}^{\max } \leq \log _{2}(n+1)$ on the maximal symmetric entanglement. The integrand of Equation (16) can be visualized by a spherical plot, and the constant integration volume can be understood as the constant volume of the plot. Figure 7(b) shows such a plot for a symmetric 12 qubit state.

Majorana representations with a high degree of symmetry are particularly easy to investigate. It is therefore elucidating to know the necessary and sufficient conditions for a rotational symmetry of the MP distribution.

Lemma 3. The MP distribution of a symmetric $n$ qubit state $|\psi\rangle_{s}$ is rotationally symmetric around the $Z$-axis 
with rotational angle $\theta=\frac{2 \pi}{m}(1<m \leq n)$ iff

$$
\forall\left\{k_{i}, k_{j} \mid a_{k_{i}} \neq 0 \wedge a_{k_{j}} \neq 0\right\}:\left(k_{i}-k_{j}\right) \bmod m=0 \text {. }
$$

This lemma states that all non-vanishing coefficients must be spaced apart from each other by a multiple of $m>1$. An example of a rotationally symmetric state with $\theta=\pi / 2$ would be $|\psi\rangle_{\mathrm{s}}=a_{3}\left|S_{3}\right\rangle+a_{7}\left|S_{7}\right\rangle+a_{15}\left|S_{15}\right\rangle$.

Symmetric states whose coefficients are all real can be associated with a reflective symmetry of the Majorana representation along the $X-Z$-plane. From a mathematical point of view two Bloch vectors $\left|\phi_{1}\right\rangle$ and $\left|\phi_{2}\right\rangle$ exhibit such a reflective symmetry iff they are complex conjugates, i.e. $\left|\phi_{1}\right\rangle=\cos \frac{\theta}{2}|0\rangle+\mathrm{e}^{\mathrm{i} \varphi} \sin \frac{\theta}{2}|1\rangle$ and $\left|\phi_{2}\right\rangle=\cos \frac{\theta}{2}|0\rangle+\mathrm{e}^{-\mathrm{i} \varphi} \sin \frac{\theta}{2}|1\rangle=\left|\phi_{1}\right\rangle^{*}$.

Lemma 4. Let $|\psi\rangle_{s}$ be a symmetric state of $n$ qubits. $|\psi\rangle_{s}$ is real iff all its MPs are reflective symmetric with respect to the $X-Z$-plane of the Majorana sphere.

It immediately follows from the form of the min-maxproblem (15) that this reflective symmetry is also inherited to the CPPs.

Particularly strong results about the number and locations of CPPs can be obtained for positive symmetric states. With the exception of the Dicke states, any positive symmetric state can have at most $2 n-4$ CPPs, and it is believed that this result also holds for general symmetric states. Dicke states are a special case due to their continuous azimuthal symmetry, resulting in an uncountable number of CPPs.

Lemma 5. Let $|\psi\rangle_{s}$ be a positive symmetric state of $n$ qubits, excluding the Dicke states.

(a) If $|\psi\rangle_{s}$ is not rotationally symmetric around the $Z$ axis, then all its CPPs are positive.

(b) If $|\psi\rangle_{s}$ is rotationally symmetric around the Z-axis with minimal rotational angle $\frac{2 \pi}{m}$, then all its CPPs $|\sigma(\theta, \varphi)\rangle=\cos \frac{\theta}{2}|0\rangle+\mathrm{e}^{\mathrm{i} \varphi} \sin \frac{\theta}{2}|1\rangle$ are restricted to the $m$ azimuthal angles given by $\varphi=\varphi_{r}=\frac{2 \pi r}{m}$ with $r \in \mathbb{Z}$. Furthermore, if $\left|\sigma\left(\theta, \varphi_{r}\right)\right\rangle$ is a $C P P$ for some $r$, then it is also a CPP for all other values of $r$.

The restriction of the CPPs to certain azimuthal angles imposed by this lemma is crucial for the rather technical proof (c.f. Appendix B of [3] ) of the following statement about the number and locations of the CPPs.

Theorem 6. The Majorana representation of every positive symmetric state of $n$ qubits, excluding the Dicke states, belongs to one of the following three classes.

(a) $|\psi\rangle_{s}$ is rotationally symmetric around the Z-axis, with only the two poles as possible CPPs.

(b) $|\psi\rangle_{s}$ is rotationally symmetric around the Z-axis, with at least one CPP being non-positive. (c) $|\psi\rangle_{s}$ is not rotationally symmetric around the $Z$ axis, and all CPPs are positive.

Regarding the CPPs of states from class (b) and (c), the following assertions can be made for $n \geq 3$ qubits:

(b) If both poles are occupied by at least one MP each, then there are at most $2 n-4$ CPPs, else there are at most $n$ CPPs.

\section{(c) There are at most $\left\lceil\frac{n+2}{2}\right\rceil$ CPPs}

The upper bound on the number of CPPs is intriguing, because the Euler characteristic implies that convex polyhedra with $n$ vertices have at most $2 n-4$ faces. One could therefore ask whether there exists a deeper relationship between the CPPs and the faces of the MP distribution.

\section{SOLUTIONS FOR UP TO TWELVE QUBITS}

An exhaustive search for the maximally entangled symmetric state over the whole space of symmetric states becomes infeasible already for only a few qubits, because the min-max-problem (15) is too intractable to easily determine solutions. The results from the previous section as well as the fact that the maximally entangled state must have at least two CPPs (c.f. Lemma 4 in [3]) considerably simplify the numerical search for high and maximal symmetric entanglement, particularly among the subset of positive symmetric states, allowing the reliable determination of the maximally entangled positive symmetric states of up to 12 qubits. For the general non-positive case an exhaustive search over the entire Hilbert space is still too involved, so we concentrated on sets of promising states. Such states include those with highly spread out MP distributions and those that share qualitative features with the solutions to the classical optimization problems. Table I summarizes the presumed values of maximal geometric entanglement for symmetric states in the positive and general case. For comparison purposes, the known upper and lower bounds are also listed. For a detailed presentation and discussion of all the solutions we refer to [3] .

For $n=2,3$ qubits the maximally entangled states were already identified as the Bell states and the $\mathrm{W}$ state, respectively. For $n=4,6,12$ the Majorana problem is solved by the respective Platonic solid, i.e. the MP distributions are given by the vertices of the corresponding Platonic solid.

The "tetrahedron state" of four qubits, shown in Figure 4, has the form $\left|\Psi_{4}\right\rangle=1 / \sqrt{3}\left|S_{0}\right\rangle+\sqrt{2 / 3}\left|S_{3}\right\rangle$. Since the state is positive and has a $\mathrm{Z}$-axis rotational symmetry, Lemma 5 restricts the CPPs to the three half-circles shown as blue lines in Figure 4 (a). By means of the tetrahedral rotation group it is possible to find a unitary operation $U \neq \mathbb{1}$ so that Equation (13) maps $\left|\Psi_{4}\right\rangle$ onto itself. This can be understood as a rotation on the Majorana sphere which moves each MP to the location of 
TABLE I. Values for the maximal entanglement of symmetric $n$ qubit states in terms of the geometric measure. The entanglement values listed are (from left to right) those of the most entangled Dicke state, the maximally entangled positive symmetric state, the presumably maximally entangled symmetric state and the upper bound on symmetric entanglement. The relation $E_{\mathrm{g}}\left(\left|S_{\lfloor n / 2\rfloor}\right\rangle\right) \leq E_{\mathrm{g}}\left(\left|\Psi_{n}^{\text {pos }}\right\rangle\right) \leq E_{\mathrm{g}}\left(\left|\Psi_{n}\right\rangle\right)<\log _{2}(n+1)$ holds for all $n$, and wherever the amount of entanglement does not increase, the respective right-hand table cell has been intentionally left blank. All numerical values have been calculated for ten or more digits, and the dagger $\dagger$ in the second column indicates values whose analytic form is known, but not displayed due to their complicated form.

\begin{tabular}{lcccc}
\hline$n$ & $E_{\mathrm{g}}\left(\left|S_{\lfloor n / 2\rfloor}\right\rangle\right)$ & $E_{\mathrm{g}}\left(\left|\Psi_{n}^{\mathrm{pos}}\right\rangle\right)$ & $E_{\mathrm{g}}\left(\left|\Psi_{n}\right\rangle\right)$ & $\log _{2}(n+1)$ \\
\hline 2 & 1 & & & $\log _{2} 3$ \\
3 & $\log _{2}(9 / 4)$ & & 2 \\
4 & $\log _{2}(8 / 3)$ & $\log _{2} 3$ & & $\log _{2} 5$ \\
5 & 1.532824877 & $1.742268948^{\dagger}$ & & 2.584962501 \\
6 & $\log _{2}(16 / 5)$ & $\log _{2}(9 / 2)$ & & $\log _{2} 7$ \\
7 & 1.767313935 & $2.298691396^{\dagger}$ & & 3 \\
8 & 1.870716983 & 2.445210159 & & 3.169925001 \\
9 & 1.942404615 & $2.553960277^{\dagger}$ & & 3.321928095 \\
10 & 2.022720077 & 2.679763092 & 2.737432003 & 3.459431619 \\
11 & 2.082583285 & 2.773622669 & 2.817698505 & 3.584962501 \\
12 & 2.148250959 & 2.993524700 & $\log _{2}(243 / 28)$ & 3.700439718 \\
\hline
\end{tabular}

another MP. A rotation of this type, with the Bloch vector of $\left|\phi_{4}\right\rangle$ acting as the rotation axis, is performed twice between Figure 4(a) and Figure 4(c). For each of these configurations Lemma 5 gives rise to separate restrictions on the locations of the CPPs, and the intersection of all these restrictions leaves only four points, the MPs them-
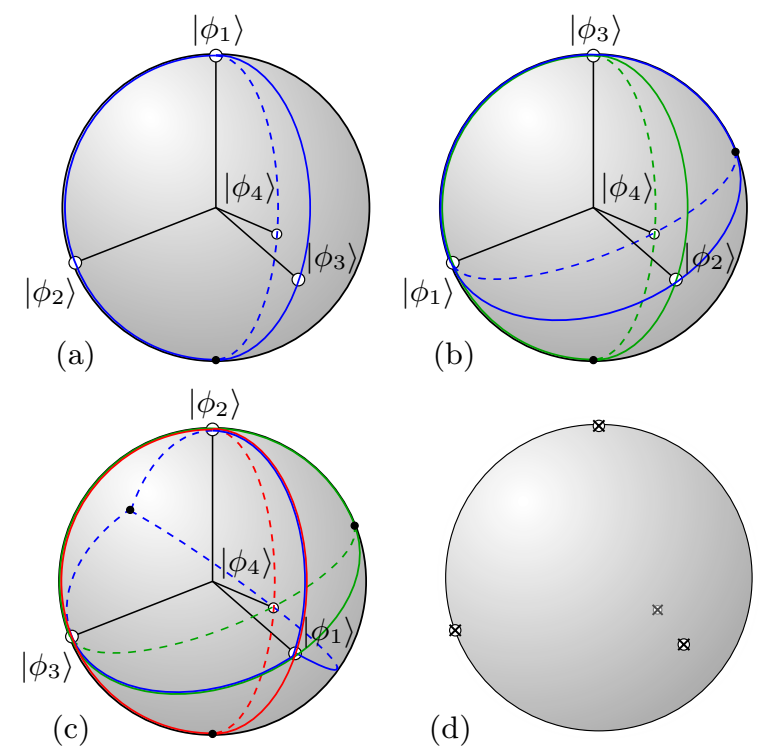

FIG. 4. (color online) The CPPs of the positive symmetric tetrahedron state $\left|\Psi_{4}\right\rangle$ of four qubits can be directly obtained from the tetrahedral rotation group and Lemma 5 Applying finite rotations amounts to permutations of the MPs and thus additional restrictions for the locations of the CPPs are obtained from Lemma 5 .
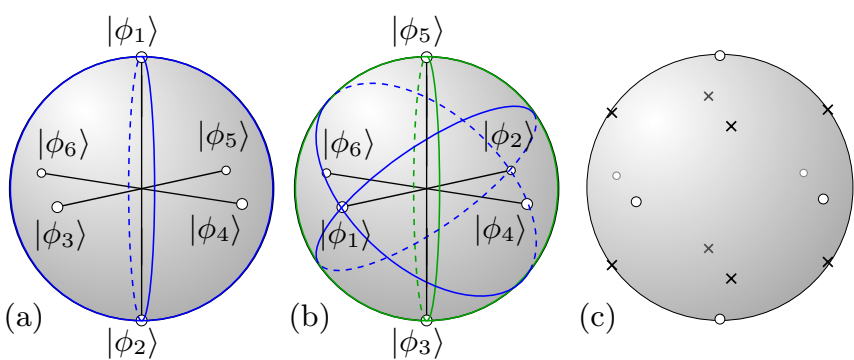

FIG. 5. (color online) Only one rotation from the octahedral rotation group is required to uniquely determine the locations of the eight CPPs of the octahedron state $\left|\Psi_{6}\right\rangle$.

selves. Therefore $\left|\Psi_{4}\right\rangle$ has four CPPs which coincide with the MPs.

For the "octahedron state" of six qubits $\left|\Psi_{6}\right\rangle=$ $1 / \sqrt{2}\left(\left|S_{1}\right\rangle+\left|S_{5}\right\rangle\right)$, shown in Figure 5 , the CPPs can be determined in the same way. Only one rotation from the octahedral rotation group is required to find the eight CPPs at the intersections of the blue and green lines depicted in Figure [5). The CPPs lie at the center of each face of the octahedron, forming a cube inside the Majorana sphere. In contrast to the tetrahedron state with its overlapping MPs and CPPs, the CPPs of the octahedron state lie as far away from the MPs as possible. This is because the expression (15) would be zero if a CPP $|\sigma\rangle$ were to lie antipodal to a MP $\left|\phi_{i}\right\rangle$.

For five points the solution to the classical problems is the trigonal bipyramid [70], and the corresponding "trigonal bipyramid state" $\left|\psi_{5}\right\rangle=1 / \sqrt{2}\left(\left|S_{1}\right\rangle+\left|S_{4}\right\rangle\right)$ is shown in Figure 6(a). This is however not the maximally entangled symmetric state, and a numerical search yields the "square pyramid state" $\left|\Psi_{5}\right\rangle \approx 0.547\left|S_{0}\right\rangle+0.837\left|S_{4}\right\rangle$, shown in Figure 6(b), as the maximally entangled one. All its MPs and CPPs can be determined analytically by solving quartic equations. One on the five CPPs coincides with the north pole while the other four are equidistantly spread over a horizontal plane in the southern hemisphere. Notably, the "center of mass" of the five MPs of $\left|\Psi_{5}\right\rangle$ does not coincide with the origin of the sphere, and the implications of this will be outlined in Section VIB

There is strong evidence that the "icosahedron state" $\left|\Psi_{12}\right\rangle=\sqrt{7}\left|S_{1}\right\rangle-\sqrt{11}\left|S_{6}\right\rangle-\sqrt{7}\left|S_{11}\right\rangle$, shown in Figure
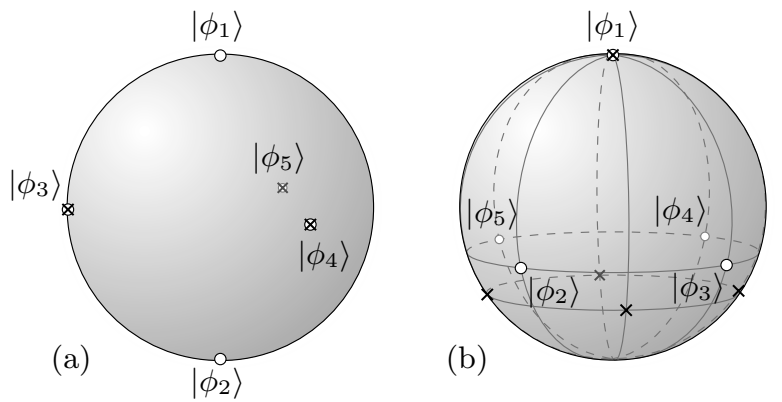

FIG. 6. The "trigonal bipyramid state" is shown in (a), but the Majorana problem of five qubits is solved by the "square pyramid state" shown in (b). 
(a)

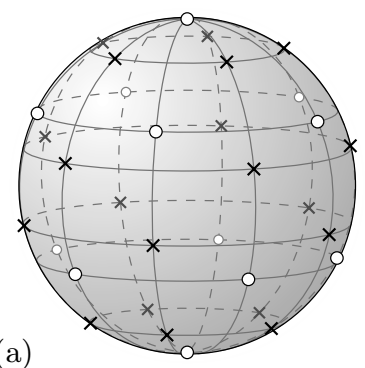

(b)

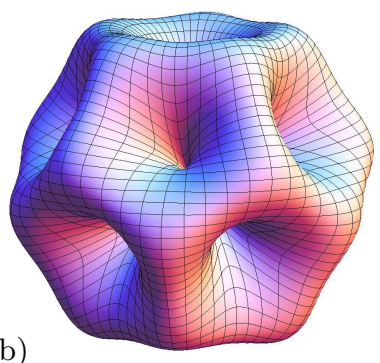

FIG. 7. (color online) The MPs and CPPs of the 12 qubit "icosahedron state" $\left|\Psi_{12}\right\rangle$ are depicted in (a), and the corresponding amplitude function $f(\theta, \varphi)=\left|\left\langle\lambda(\theta, \varphi) \mid \Psi_{12}\right\rangle\right|$ is shown in (b). For $\left|\Psi_{12}\right\rangle$ the locations of the MPs and CPPs coincide with the zeros and maxima of $f(\theta, \varphi)$, respectively.

7(a), is the maximally entangled symmetric state of 12 qubits. The MPs form the vertices of a regular icosahedron, while the 20 CPPs are centered on the faces of the icosahedron, describing a dodecahedron inside the Majorana sphere. Figure $7(\mathrm{~b})$ is the spherical plot of the function $f(\theta, \varphi)=\left|\left\langle\lambda(\theta, \varphi) \mid \Psi_{12}\right\rangle\right|$ which already appeared as the integrand of Equation (16). This function is variously known as the characteristic polynomial, Majorana polynomial [26], amplitude function 71] or coherent state decomposition [25]. The CPPs and MPs of a symmetric state can be readily identified as the global maxima and the antipodes of the zeros of $f(\theta, \varphi)$, respectively.

\section{A. Dual Polyhedra}

Each of the five Platonic solids shown in Figure 2 has a dual polyhedron with faces and vertices interchanged, and this dual polyhedron is again a Platonic solid 32]. As seen in Figure 8, the octahedron and cube form a dual pair, and so do the icosahedron and dodecahedron. In contrast to this, the tetrahedron is self-dual, i.e. it is its own dual.

Interestingly, these dualities are also inherited to the Majorana representations of the corresponding symmetric quantum states. For example, we have seen that the 20 CPPs of the icosahedron state $\left|\Psi_{12}\right\rangle$ form the vertices of a dodecahedron. On the other hand, when considering the 20 qubit "dodecahedron state" $\left|\Psi_{20}\right\rangle=\sqrt{187}\left|S_{0}\right\rangle+$ $\sqrt{627}\left|S_{5}\right\rangle+\sqrt{247}\left|S_{10}\right\rangle-\sqrt{627}\left|S_{15}\right\rangle+\sqrt{187}\left|S_{20}\right\rangle$, it is easy to show that this state has 12 CPPs which occupy the
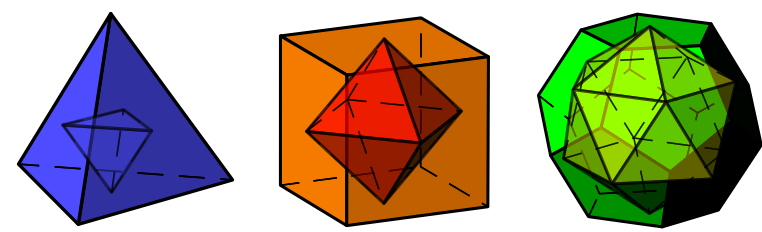

FIG. 8. (color online) The relationships between the Platonic solids and their duals. vertices of an icosahedron. Thus the Majorana representation of the dodecahedron state can be immediately obtained from Figure 7(a) by interchanging the MPs and CPPs. The same duality exists between the octahedron state and the cube state, c.f. Figure 5 (c). Furthermore, the tetrahedron state is its own dual, as seen in Figure 4 (d). Unlike the dual of the Platonic solid, however, the dual tetrahedron state is not turned "upside down" as seen in Figure 8, but rather coincides with the original tetrahedron state.

\section{B. Anticoherent Spin States and the Queens of Quantum}

As outlined in Section IV, there exists an isomorphism between the states of a spin- $j$ particle and the symmetric states of $2 j$ qubits. The coherent states of a quantum particle can be regarded as the most classical states, and in terms of the Majorana representation these states are those whose MPs all coincide at a single point, thus describing a "classical" spin vector. Anticoherent spin states, first studied in [33], are states that exhibit maximally nonclassical behavior in the sense that their spin vector vanishes. Since such states can be considered the "opposite" of coherent states, it would be interesting to determine the MPs and the geometric entanglement of their symmetric counterparts. For example, one could ask whether maximally entangled symmetric states correspond to anticoherent states or to the mathematical concept of spherical designs [22]. However, the fact that the "center of mass" of the five qubit square pyramid state $\left|\Psi_{5}\right\rangle$ does not coincide with the origin of the Majorana sphere straightforwardly implies that this state is neither anticoherent nor a spherical design [72].

An alternative to anticoherent states was formulated in [21], where the least classical states are coined "queens of quantum". The Majorana representations of these states differ from our maximally entangled symmetric states, but when replacing the Hilbert-Schmidt metric with the Bures metric [4], the solutions of the two problems become identical. In other words, the Majorana representation of the spin- $j$ "queen of quantum" in terms of the Bures metric is identical to that of the maximally entangled symmetric state of $2 j$ qubits in terms of the geometric measure.

\section{CONCLUSION}

We have analyzed and discussed the geometric entanglement of highly and maximally entangled symmetric states of $n$ qubits. The upper bound on symmetric entanglement rules out symmetric states as exact, deterministic MBQC resources. For the case of approximate MBQC we present arguments against the usefulness of symmetric states, and provide a proof for the class of Dicke states. With the known analytic results about the 
Majorana representation of symmetric states it is easy to numerically determine the most entangled states and to discuss their properties. As an example we showed how the determination of the CPPs of "Platonic states" is greatly simplified with the help of the theoretical results. With the help of the maximally entangled symmetric five qubit state it was shown that the solutions to the Majorana problem do not necessarily relate to anticoherent states or spherical designs. It is found that the well-known concept of the dual polyhedra of Platonic solids possesses a direct analog for symmetric quantum states, thereby deepening the relationship between the Majorana representation and the polyhedra of classical

[1] W. Dür, G. Vidal, and J. I. Cirac, Phys. Rev. A 62, 062314 (2000).

[2] R. Horodecki, P. Horodecki, M. Horodecki, and K. Horodecki, Rev. Mod. Phys. 81, 865 (2009).

[3] M. Aulbach, D. Markham, and M. Murao, New J. Phys. 12, 073025 (2010).

[4] J. Martin, O. Giraud, P. A. Braun, D. Braun, and T. Bastin, Phys. Rev. A 81, 062347 (2010).

[5] E. D'Hondt and P. Panangaden, Quant. Inf. Comp. 6, 173 (2006).

[6] R. Prevedel et al., Phys. Rev. Lett. 103, 020503 (2009).

[7] W. Wieczorek et al., Phys. Rev. Lett. 103, 020504 (2009).

[8] T. Bastin et al., Phys. Rev. Lett. 103, 070503 (2009).

[9] M. Hayashi, D. Markham, M. Murao, M. Owari, and S. Virmani, Phys. Rev. A 77, 012104 (2008).

[10] R. Hübener, M. Kleinmann, T.-C. Wei, C. GonzálezGuillén, and O. Gühne, Phys. Rev. A 80, 032324 (2009).

[11] D. Markham, arXiv:1001.0343 (2010).

[12] P. Mathonet et al., Phys. Rev. A 81, 052315 (2010).

[13] G. Tóth and O. Gühne, Phys. Rev. Lett. 102, 170503 (2009).

[14] M. Van den Nest, A. Miyake, W. Dür, and H. J. Briegel, Phys. Rev. Lett. 97, 150504 (2006).

[15] D. Gross, S. T. Flammia, and J. Eisert, Phys. Rev. Lett. 102, 190501 (2009).

[16] C. E. Mora et al., Phys. Rev. A 81, 042315 (2010).

[17] M. Van den Nest, W. Dür, A. Miyake, and H. J. Briegel, New J. Phys. 9, 204 (2007).

[18] E. Majorana, Nuovo Cimento 9, 43 (1932).

[19] M. Aulbach and D. Markham, (in preparation).

[20] R. Barnett, A. Turner, and E. Demler, Phys. Rev. A 76, 013605 (2007).

[21] O. Giraud, P. A. Braun, and D. Braun, New J. Phys. 12, 063005 (2010).

[22] J. Crann, R. Pereira, and D. W. Kribs, J. Phys. A: Math. Theo. 43, 255307 (2010).

[23] J. H. Hannay, J. Phys. A: Math. Gen. 29, L101 (1996).

[24] J. H. Hannay, J. Phys. A: Math. Gen. 31, L53 (1998).

[25] P. Leboeuf, J. Phys. A: Math. Gen. 24, 4575 (1991).

[26] P. Kolenderski and R. Demkowicz-Dobrzanski, Phys. Rev. A 78, 052333 (2008). geometry.

\section{ACKNOWLEDGMENTS}

The authors would like to thank S. Miyashita, A. Soeda, S. Virmani, K.-H. Borgwardt and M. Van den Nest for very helpful discussions. This work is supported by the National Research Foundation \& Ministry of Education, Singapore and the project "Quantum Computation: Theory and Feasibility" in the framework of the CNRS-JST Strategic French-Japanese Cooperative Program on ICT. MM thanks the "Special Coordination Funds for Promoting Science and Technology" for financial support.
[27] P. Kolenderski, Open Systems \& Information Dynamics 17, 107 (2009).

[28] H. Mäkelä and K.-A. Suominen, Phys. Rev. Lett. 99, 190408 (2007).

[29] D. Markham and V. Vedral, Phys. Rev. A 67, 042113 (2003).

[30] P. Ribeiro, J. Vidal, and R. Mosseri, Phys. Rev. E 78, 021106 (2008).

[31] J. Zimba and R. Penrose, Stud. Hist. Phil. Sci. 24, 697 (1993).

[32] M. J. Wenninger, Dual Models (Cambridge University Press, 1983).

[33] J. Zimba, Electron. J. Theor. Phys. 3, 143 (2006).

[34] V. Vedral and M. B. Plenio, Phys. Rev. A 57, 1619 (1998).

[35] H. Barnum and N. Linden, J. Phys. A: Math. Gen. 34, 6787 (2001).

[36] A. Shimony, Ann. NY. Acad. Sci. 755, 675 (1995).

[37] T.-C. Wei and P. M. Goldbart, Phys. Rev. A 68, 042307 (2003).

[38] G. Vidal and R. Tarrach, Phys. Rev. A 59, 141 (1999).

[39] D. Cavalcanti, Phys. Rev. A 73, 044302 (2006).

[40] T.-C. Wei, M. Ericsson, P. M. Goldbart, and W. J. Munro, Quant. Inf. Comp. 4, 252 (2004).

[41] M. Hayashi, D. Markham, M. Murao, M. Owari, and S. Virmani, Phys. Rev. Lett. 96, 040501 (2006).

[42] D. Markham, A. Miyake, and S. Virmani, New J. Phys. 9, 194 (2007)

[43] L. De Lathauwer, B. De Moor, and J. Vandewalle, SIAM J. Matrix Anal. Appl. 21, 1324 (2000).

[44] G. Ni and Y. Wang, Math. Comput. Modelling 46, 1345 (2007).

[45] V. De Silva and L. H. Lim, SIAM J. Matrix Anal. Appl. 30, 1084 (2008).

[46] R. F. Werner and A. S. Holevo, J. Math. Phys. 43, 4353 (2002).

[47] H. Zhu, L. Chen, and M. Hayashi, New J. Phys. 12, 083002 (2010).

[48] R. H. Dicke, Phys. Rev. 93, 99 (1954).

[49] G. Tóth, J. Opt. Soc. Am. B 24, 275 (2007).

[50] J. K. Korbicz, J. I. Cirac, and M. Lewenstein, Phys. Rev. Lett. 95, 120502 (2005).

[51] J. K. Korbicz et al., Phys. Rev. A 74, 052319 (2006). 
[52] R. Orús, S. Dusuel, and J. Vidal, Phys. Rev. Lett. 101, 025701 (2008).

[53] M. B. Plenio and S. Virmani, Quant. Inf. Comp. 7, 1 (2007)

[54] E. Jung et al., Phys. Rev. A 77, 062317 (2008).

[55] R. Renner, PhD thesis, ETH Zurich, 2005, arXiv:quant-ph/0512258

[56] There exist two common definitions of the geometric measure, which we distinguish by denoting $E_{\mathrm{g}}(|\psi\rangle)=$ $-\log _{2}\left|\left\langle\Lambda_{\psi} \mid \psi\right\rangle\right|^{2}$ and $E_{\mathrm{G}}(|\psi\rangle)=1-\left|\left\langle\Lambda_{\psi} \mid \psi\right\rangle\right|^{2}$. The former is used throughout this article, with the only exception being this subsection, where $E_{\mathrm{G}}$ is used in order to be consistent with the notation of [16].

[57] H. Bacry, J. Math. Phys. 15, 1686 (1974).

[58] S. Tamaryan, T.-C. Wei, and D. Park, Phys. Rev. A 80, 052315 (2009).

[59] L. Chen, A. Xu, and H. Zhu, Phys. Rev. A 82, 032301 (2010).

[60] L. L. Whyte, Am. Math. Mon. 59, 606 (1952).
[61] J. J. Thomson, Phil. Mag. 7, 237 (1904)

[62] P. Leiderer, Z. Phys. B 98, 303 (1995).

[63] E. J. Davis, Aerosol Sci. Technol. 26, 212 (1997)

[64] C. J. Marzec and L. A. Day, Biophys. J. 65, 2559 (1993).

[65] A. D. Dinsmore et al., Science 298, 1006 (2002).

[66] H. W. Kroto, J. R. Heath, S. C. O'Brien, R. F. Curl, and R. E. Smalley, Nature 318, 162 (1985).

[67] M. J. W. Dodgson and M. A. Moore, Phys. Rev. B 55, 3816 (1997).

[68] T. Erber and G. M. Hockney, J. Phys. A: Math. Gen. 24, L1369 (1991).

[69] E. L. Altschuler, T. J. Williams, E. R. Ratner, F. Dowla, and F. Wooten, Phys. Rev. Lett. 72, 2671 (1994).

[70] N. Ashby and W. E. Brittin, Am. J. Phys. 54, 776 (1986).

[71] J. M. Radcliffe, J. Phys. A: Math. Gen. 4, 313 (1971).

[72] For anticoherent spin states this readily follows from $\left\langle\Psi_{5}\left|S_{z}\right| \Psi_{5}\right\rangle \neq 0$. For spherical designs we observe that by setting $p(x)=x$ in Definition 2 of [22], it follows that for all spherical designs the "center of mass" must necessarily coincide with the sphere's origin. 